\title{
Life Satisfaction Shows Terminal Decline in Old Age: Longitudinal Evidence From the German Socio-Economic Panel Study (SOEP)
}

\author{
Denis Gerstorf and Nilam Ram \\ Pennsylvania State University and Max Planck Institute for \\ Human Development \\ Jürgen Schupp \\ DIW Berlin (German Institute for Economic Research) and Free \\ University of Berlin
}

\author{
Ryne Estabrook \\ University of Virginia
}

Gert G. Wagner

DIW Berlin (German Institute for Economic Research) and Berlin University of Technology

Ulman Lindenberger

Max Planck Institute for Human Development

\begin{abstract}
Longitudinal data spanning 22 years, obtained from deceased participants of the German SocioEconomic Panel Study (SOEP; $N=1,637 ; 70$ - to 100-year-olds), were used to examine if and how life satisfaction exhibits terminal decline at the end of life. Changes in life satisfaction were more strongly associated with distance to death than with distance from birth (chronological age). Multiphase growth models were used to identify a transition point about 4 years prior to death where the prototypical rate of decline in life satisfaction tripled from -0.64 to $-1.94 T$-score units per year. Further individual-level analyses suggest that individuals dying at older ages spend more years in the terminal periods of life satisfaction decline than individuals dying at earlier ages. Overall, the evidence suggests that late-life changes in aspects of well-being are driven by mortality-related mechanisms and characterized by terminal decline.
\end{abstract}

Keywords: selective mortality, successful aging, multiphase growth models, psychosocial factors, wellbeing

Lifespan psychological and gerontological researchers have long been interested in phenomena of terminal decline (Birren \& Cunningham, 1985; Busse, 1969; Kleemeier, 1962; Palmore \&

Denis Gerstorf and Nilam Ram, Department of Human Development and Family Studies, Pennsylvania State University, and Max Planck Institute for Human Development, Berlin, Germany; Ryne Estabrook, Department of Psychology, University of Virginia; Jürgen Schupp, German Socio-Economic Panel Study, DIW Berlin (German Institute for Economic Research), Berlin, Germany, and Free University of Berlin, Berlin, Germany; Gert G. Wagner, German Socio-Economic Panel Study, DIW Berlin (German Institute for Economic Research) and Berlin University of Technology, Berlin, Germany; Ulman Lindenberger, Max Planck Institute for Human Development.

Parts of this article were prepared while Denis Gerstorf was at the Department of Psychology, University of Virginia, on a research fellowship awarded by the German Research Foundation. This research was supported in part by National Institute on Aging T32 Grant AG20500-01 to Ryne Estabrook and Bundesministerium für Bildung und Forschung (Germany) Grant DIW001 to Jürgen Schupp and Gert G. Wagner. The German Socio-Economic Panel Study is financed by the Wissenschaftsgemeinschaft Gottfried Wilhelm Leibniz.

Correspondence concerning this article should be addressed to Denis Gerstorf, Department of Human Development and Family Studies, Pennsylvania State University, 118 Henderson Building, University Park, PA 16802. E-mail: gerstorf@psu.edu
Cleveland, 1976; Riegel \& Riegel, 1972; Siegler, 1975). The general notion is that at some point shortly before death, individuals' functioning declines quite rapidly. In various cognitive domains, evidence is building that late-life changes in function are marked by pronounced deteriorations proximate to death (for review, see Bäckman \& MacDonald, 2006). Only recently, however, have researchers begun to examine how notions of terminal decline may apply to other aspects of psychological function such as well-being (Gerstorf, Ram, Röcke, Lindenberger, \& Smith, 2008; Mroczek \& Spiro, 2005). The present study uses 22-year longitudinal data from the nationally representative German SocioEconomic Panel Study to examine questions about terminal decline in old age in life satisfaction, a key component of well-being. Specifically, we (a) examine whether changes in life satisfaction that occur in old age are better characterized as age-related or mortality-related processes, (b) use multiphase growth models to articulate and test notions of terminal decline, and (c) explore whether the onset of terminal decline may differ with age at death, sex, and education.

An accumulating body of empirical evidence suggests that low levels of functioning and pronounced decline on a number of psychosocial factors, including cognitive functioning and wellbeing, are predictive of subsequent mortality (e.g., Bosworth \& Schaie, 1999; Danner, Snowdon, \& Friesen, 2001; Ghisletta, McArdle, \& Lindenberger, 2006; Levy, Slade, Kunkel, \& Kasl, 
2002; Maier \& Smith, 1999; White \& Cunningham, 1988). When viewed next to evidence that well-being remains relatively stable throughout adulthood and old age (Diener, Lucas, \& Scollon, 2006; Kunzmann, Little, \& Smith, 2000; Mroczek \& Kolarz, 1998), unique associations between well-being and mortality seem paradoxical. One interpretation is that late-life changes in wellbeing may primarily be driven by mortality-related processes rather than normative age-related processes. The underlying idea is that individuals typically have enough resources to maintain a sense of well-being even as they face increased risks for losses in the social and health domains. As death approaches, however, individuals are faced with additional mortality-related burdens that make it increasingly difficult to maintain well-being-and the system collapses. If this speculation is true, intraindividual changes in well-being that occur in the last years of life would be more closely associated to distance to death rather than distance from birth (i.e., chronological age).

Notions of terminal decline, as most often presented in the cognitive domain (for review, see Bäckman \& MacDonald, 2006), predict a multiphase sequence of change. Individuals transition from a preterminal phase of normative gradual decline in functionality to a terminal phase of pronounced decline when the burdens of an approaching death begin to overwhelm a limited pool of resources (Kleemeier, 1962; Sliwinski et al., 2006; Thorvalddon, Hofer, \& Johansson, 2006). Recently, empirical investigations of terminal decline phenomena have taken advantage of methodological innovations that allow fitting of growth curve models that articulate with specificity if and how intraindividual changes are characterized by multiple phases of change (for discussion of multiphase growth models, see Cudeck \& Harring, 2007; Cudeck \& Klebe, 2002; Hall, Lipton, Sliwinski, \& Stewart, 2000; Ram \& Grimm, 2007). In the cognitive domain, for example, multiphase growth models have been used to identify multiple phases of mortality-related decline in perceptual speed and memory (Sliwinski et al., 2006; Wilson, Beck, Bienias, \& Bennett, 2007; Wilson, Beckett, Bienias, Evans, \& Bennett, 2003). Extending the approach to aspects of well-being, Gerstorf et al. (2008) provided initial evidence that late-life intraindividual changes in life satisfaction are also characterized by terminal decline trajectories. More specifically, terminal-decline representations of change, characterized by a multiphase model with a discrete shift to a twofold increase in steepness of decline at about $4( \pm 2.5)$ years prior to death, were found to provide for more efficient descriptions of the data than did linear representations over both chronological age and distance to death. In the absence of other studies on terminal decline of life satisfaction, the purpose of the present study is to further describe the phenomena and to replicate and extend those initial findings.

The terminal decline hypothesis makes specific predictions that intraindividual changes can be structured along a transition from a preterminal phase of normative gradual decline into a terminal phase of pronounced decline shortly before death. However, the theory on terminal decline, in our reading, has remained vague about when the transition to terminal decline should typically occur (e.g., Kleemeier, 1962; Palmore \& Cleveland, 1976; Riegel \& Riegel, 1972; Siegler, 1975). The limited evidence from studies of terminal decline in cognition and life satisfaction has located transition points in a window ranging from 2 to 6 years (Gerstorf et al., 2008; Wilson et al., 2003, 2007) or even 8 years (Sliwinski et al., 2006) prior to death. The present study contributes to this exploration. Using a nationally representative sample and multiphase growth models where the point of transition is estimated directly from the data, we examined when in relation to death a terminal phase of decline in life satisfaction may begin.

It is largely an open question whether men and women or individuals who die at earlier ages versus later ages experience the last years of life differently. Consistent with the view that selfprotective processes become increasingly vulnerable as individuals get older (Baltes \& Smith, 2003), there is some evidence to suggest steeper mortality-related declines with greater age of death (Bäckman \& MacDonald, 2006; Gerstorf et al., 2008). It also appears that individual difference characteristics such as high educational attainment, low comorbidities, and preserved cognitive functioning may provide protective resources against late-life functional decline. However, Gerstorf et al. (2008) reported that all of these well-established mortality predictors accounted for only a very small portion of interindividual differences in terminal change in life satisfaction. The present study reexamines such relationships in the context of participants drawn from a nationally representative study. Specifically, we note if and how age at death, sex, and education are associated with individual differences in mortalityrelated life satisfaction changes. Although we do not have specific hypotheses as to potential sex and education differences, we expect that impending mortality has more detrimental effects on functionality at older ages.

To summarize, the present study attempts to replicate and extend recent findings on mortality-related changes in life satisfaction in old age. We (a) determine whether mortality-related (distance to death) models of late-life changes in life satisfaction provide better representations of the observed changes in old age than do age-related models; (b) use multiphase growth models to articulate terminal decline hypotheses and derive an empirically based location for the transition into terminal decline of life satisfaction; and (c) examine if and how the changes in life satisfaction that occur at the end of life differ with age at death, sex, and education.

\section{Method}

Growth curve models were fitted to 22 waves of yearly (19842005) longitudinal data from now-deceased, 70- to 100-year-old participants $(N=1,637)$ in the German Socio-Economic Panel Study (SOEP) to examine intraindividual change in life satisfaction as a function of age or distance to death. Descriptions of the larger SOEP study and its design, participants, variables, and assessment procedures can be found in Haisken-De New and Frick (2006) and Wagner, Frick, and Schupp (2007). Select details relevant to the present study are given below.

\section{Sample and Participant Selection}

The SOEP is a nationally representative longitudinal annual panel study of private households and individuals. In total, the SOEP data currently span 22 years, 1984-2005, and have a participant base of roughly 22,000 persons, including residents of former West and East Germany, immigrants, and resident foreigners. When recruited in 1984, 1990, 1995, 1998, 2000, or 2002, participants were drawn at random from a set of randomly selected 
locations within Germany. Response rates were sufficiently high (between $60 \%$ and $70 \%$ ) that the demographic characteristics of the total sample are representative of the wider population living in private households of Germany (Haisken-De New \& Frick, 2006). Longitudinal attrition has been relatively low (4\%-14\% yearly attrition across various subsamples), in part due to small rewards for continued participation (e.g., information about results) and efforts to maintain regular contact with participants, including those who had missed one or more of the yearly assessments. For example, for those who started in 1984 the overall retention rate over 22 years was still at about $43 \%$ (for details, see Kroh \& Spie $\beta$, 2006). Data were collected via face-to-face interviews or, for about $10 \%$ of respondents who had already participated multiple times, via self-administered questionnaires.

For the purposes of the present gerontologically oriented study, we used data collected from 1,637 participants (727 men, 910 women) who (a) were age 70 or older at one or more assessments and (b) have since died. Specifically, of the total $\sim 22,000$ SOEP participants, 3,519 individuals $(\sim 16 \%)$ provided data points after age 70 . Of these, we selected for our analyses participants whose death prior to 2005 had been verified. These 1,637 select participants (decedents) were born between 1888 and 1935 and died, on average, 81.82 years later ( $S D=6.54$; range: $71-101$ years). They participated in an average of $7.61(S D=5.68)$ annual surveys with $n=980$ or $60 \%$ contributing five or more data points. On average, deaths occurred 9.34 years ( $S D=5.63$; range: $1-22$ years $)$ after their initial assessment and 1.73 years $(S D=2.13$; range: $0-15$ years $)$ after the last assessment in which they took part. Participants contributed a total of 10,162 observation points that simultaneously span the 70 - to 100 -year-old age range $(M=78.20, S D=5.88)$ and the corresponding 22-0 years $(M=5.58, S D=3.99)$ prior to death.

\section{Measures}

Life satisfaction. As part of the yearly and primarily economic survey, individuals responded to the question, "Wie zufrieden sind Sie gegenwärtig, alles in allem, mit ihrem Leben?" ("How satisfied are you with your life, all things considered?") on a 0 (totally unsatisfied) to 10 (totally satisfied) scale. As a reflection about life as a whole, answers are taken as an indication of life satisfaction (cf. Fujita \& Diener, 2005) and considered an assessment of cognitive-evaluative (rather than emotional) aspects of wellbeing. ${ }^{1}$ Life satisfaction scores were, on average, $6.66(S D=2.31$; range $=0-10)$, indicating that on the vast majority of occasions $(8,669$ of 10,$162 ; 85 \%)$ the older and now deceased participants in the present study reported their life satisfaction to be at or above neutral. We standardized life satisfaction scores to a $T$ metric $(M$ $=50, S D=10)$ using the entire SOEP longitudinal sample as the reference frame $(M=7.02, S D=1.55$; see Lucas, Clark, Georgellis, \& Diener, 2003). For comparison, a neutral response on the original 11-point Likert-type scale would equal 37 on our $T$-unit scale. Further details of the life satisfaction item as used in the SOEP and its measurement properties can be found in Fujita and Diener (2005), Kroh (2006), Schimmack, Schupp, and Wagner (in press), and Schilling (2006).

Time metrics of age and distance to death. Age at each assessment wave was taken as the number of years since an individual's birth (centered at 85 years). Mortality status and year of death for deceased participants was obtained either (a) by interviewers at the yearly assessments (i.e., from household members or, in the case of one-person households, neighbors) or (b) from city registries and other authorities (comprehensive database information was obtained most recently in 2001; see Infratest Sozialforschung, 2002). Comparisons of death rates and ages of death in the SOEP with those obtained from official life tables suggest that the sample is representative of German adult mortality. Similarly and in line with other reports (Rendtel, 1995; Schräpler, 2004), substituting interviewers over time or other field-related circumstances were found to be by far the more important predictors of nonresponse or dropout compared with age-related selectivity. The longitudinal sample can thus be considered unbiased with respect to age and serves as a widely accepted basis for mortality analyses in Germany (e. g., Brockmann \& Klein, 2004; Burkhauser, Giles, Lillard, $\&$ Schwarze, 2005). For data protection reasons, the timing of individual deaths was recorded by year (rather than by day and month). Distance to death (DtD) was calculated post hoc as the difference between the date of the assessment and the participant's death year. Additional demographic variables include age at death, sex, and years of formal education.

\section{Data Analysis and Structure}

To address our research questions, we evaluated and compared relative fits of single and multiphase growth models of changes in life satisfaction across age and distance-to-death time dimensions. In follow-up analyses, we explored interindividual differences in terminal decline with respect to individual difference characteristics including age at death, sex, and education.

Age-related versus mortality-related and linear versus multiphase representations of change. The main analytic task was to determine which time dimension, age or distance-to-death, provided for a better representation of the observed longitudinal changes in life satisfaction. Two sets of growth curve (i.e., multilevel) models were used to model interindividual differences in change over time (McArdle \& Nesselroade, 2003; Singer \& Willett, 2003). In the first set of models, age was used as the time metric, effectively modeling interindividual differences in how each individual's life satisfaction changed from age 70 to age 100 . In the second set of models, distance-to-death was used as the time metric, modeling how life satisfaction changed in relation to impending mortality (i.e., over the 22 years approaching death). Within each model set, linear and multiphase representations of intraindividual change were fitted, the former being used to represent continuous trajectories and the latter to represent discrete shifts in the rate of change that occur at a specific age or distance to death. Comparing the relative fit of these models, we determined the time metric and type of trajectory that best described the data.

The linear model was specified as

$$
l s_{i t}=b_{0 i}+b_{1 i}\left(\text { time }_{i t}\right)+e_{i t},
$$

\footnotetext{
${ }^{1}$ Note that studies of age-related changes in well-being have either focused on emotional aspects of well-being (e.g., positive and negative affect; Charles et al., 2001; Mroczek \& Kolarz, 1998), cognitive-evaluative aspects (e.g., Gerstorf, Lövdén, Röcke, Smith, \& Lindenberger, 2007), or both emotional and cognitive aspects (e.g., Lucas \& Gohm, 2000). Due to constraints of the data, we examined only cognitive-evaluative aspects. It would be instructive to examine whether more emotion-based measures of well-being would yield similar results to those reported in this study.
} 
where person $i$ 's reported life satisfaction at time $t, l s_{i t}$, is a function of an individual-specific intercept parameter, $b_{O i}$, an individual-specific slope parameter, $b_{1 i}$, that captures the rate of change over the selected time dimension (age or distance to death), and residual error, $e_{i t}$. Following standard multilevel or latent growth modeling procedures (see Ram \& Grimm, 2007; Raudenbush \& Bryk, 2002; Singer \& Willett, 2003), individual-specific intercepts, $b_{O i}$, and linear slopes, $b_{1 i}$ (from the Level 1 model given in Equation 1), were modeled as

$$
\begin{gathered}
b_{0 i}=a_{00}+u_{0 i}, \text { and } \\
b_{1 i}=a_{01}+u_{1 i},
\end{gathered}
$$

(i.e., Level 2 model) where $a_{O O}$ and $a_{O I}$ are sample means and $u_{O i}$ and $u_{1 i}$ are individual deviations from those means. These interindividual differences are assumed to be normally distributed, correlated with each other, and uncorrelated with the residual errors, $e_{i t}$.

The multiphase model extended the linear model to include a second slope parameter, $b_{2 i}$, and a point of transition or change point, $k$ (see also Cudeck \& Harring, 2007; Cudeck \& Klebe, 2002; Singer \& Willett, 2003). The model was specified as

$$
\begin{gathered}
l s_{i t}=b_{0 i}+b_{1 i}\left(\text { time }_{i t}-k\right)+e_{i t}, \text { when } \text { time }_{i t}<k, \text { and } \\
l s_{i t}=b_{0 i}+b_{2 i}\left(\text { time }_{i t}-k\right)+e_{i t}, \text { when } \text { time }_{i t} \geq k,
\end{gathered}
$$

where individual-specific rates of change before the transition or change point $k$ (e.g., preterminal phase) are captured by $b_{1 i}$, and individual-specific rates of change falling after the change point (e.g., terminal phase) are captured by $b_{2 i}$. The point of transition from one phase to the other, $k$, is a free (fixed-effect) parameter estimated from the data, with $b_{O i}$ capturing the estimated level of life satisfaction at this point in time. As in the linear model, interindividual differences were modeled using Level 2 equations where $u_{O i}, u_{1 i}$, and $u_{2 i}$ are assumed to be normally distributed, correlated with each other, and uncorrelated with the residual errors, $e_{i t}$. Models were fit to the data using SAS (Proc Mixed and Proc NLMixed; Littell, Miliken, Stoup, \& Wolfinger, 1996). Of interest was (a) whether age or distance-to-death models provided for a better representation of the data; and (b) whether there was evidence for multiple phases of decline (i.e., better overall fit to the data for the multiphase models relative to the linear models) and at what age or distance to death the transition between phases (i.e., the change point, $k$ ) might occur.

Interindividual differences. One constraint of the multiphase model given above is that the change-point, $k$, is fixed (assumed) to be the same for all individuals. Theoretically, however, individuals may transition into the terminal-decline phase at different times, some individuals 2 years before death, some 4 years before death, etc. (cf. Baltes \& Smith, 2003). As an initial exploration into whether such interindividual differences in the onset of terminal decline can be identified, an expanded model was fit to a subset of the data (i.e., the $n=400$ participants providing the most longitudinal data, $\sim 12+$ observations). Specifically, $k$ was reconceptualized as a random effect, $k_{i}$, in the within-person Level 1 model,

$$
\begin{aligned}
& l s_{i t}=b_{0 i}+b_{1 i}\left(\text { time }_{i t}-k_{i}\right)+e_{i t} \text {, when time } e_{i t}<k_{i} \text {, and } \\
& l s_{i t}=b_{0 i}+b_{2 i}\left(\text { time }_{i t}-k_{i}\right)+e_{i t}, \text { when } \text { time }_{i t} \geq k_{i},
\end{aligned}
$$

with interindividual differences in the change point, $k_{i}$, being modeled, along with $b_{O i}, b_{1 i}$, and $b_{2 i}$, at Level 2 . Of interest was the extent of individual differences in the point of transition to terminal decline (i.e., variance of $k_{i}$ ). Statistical estimation was conducted via Gibbs sampling in WinBugs (Spiegelhalter, Thomas, Best, \& Lunn, 2007; see also Wang \& McArdle, 2008; Zhang, Hamagami, Wang, Grimm, \& Nesselroade, 2007).

Interindividual differences in the location of the change point $\left(k_{i}\right)$ were then modeled as a function of individuals' demographic variables. Of interest was whether and how the modeled interindividual differences in the location of the change point were related to interindividual differences in age at death, sex, education, and their interactions, e.g.,

$$
\begin{aligned}
& k_{i}=a_{03}+a_{13}(\text { age at death })+a_{23}\left(\text { sex }_{i}\right)+a_{33}\left(\text { education }_{i}\right) \\
& +a_{43}\left(\text { age at death } \text { deducation }_{i}\right) \\
& +a_{53}\left(\text { age at death }{ }_{i} \times \operatorname{sex}_{i}\right)+a_{63}\left(\operatorname{sex}_{i} \times \text { education }_{i}\right) \\
& +a_{73}\left(\text { age at death }{ }_{i} \times \operatorname{sex}_{i} \times \text { education }_{i}\right)+u_{3 i} .
\end{aligned}
$$

Data structure. Descriptive statistics for life satisfaction are provided in Table 1, both over age (Columns 1-4) and distance to death (Columns 5-8). It appears that average levels of life satisfaction decrease with both age and proximity to death (e.g., $M=$ 50.23 at 20 years prior to death, $M=41.14$ at the year prior to death). It may also be noted from Table 1 that the large majority of observations were obtained when participants were age 70 to 89 or in the 10- to 0 -year period prior to death. To illustrate, $92 \%(n=$ 1,514) of our participants provided one or more data points in the last 4 years of life. We also note the primarily longitudinal nature of the data, with $88 \%(n=1,447)$ of the participants contributing information about intraindividual change by providing two or more data points $(M$ observation period $=6.13$ years, $S D=5.16$; range: $0-21$ years). Following the accelerated longitudinal design, aligning all of these segments and treating them as a single sample allowed for estimation, under missing-at-random assumptions (Little \& Rubin, 1987), of an age gradient spanning 30 years (70-100) and a distance-to-death gradient of up to 22 years. During estimation, change effects (i.e., parameters associated with age or DtD) are derived from all the longitudinal segments, with more weight being implicitly given to information obtained from those individuals who provided the most change (i.e., most occasions) information. Finally, the correlation between age and distance to death was of moderate size $(r=.27, p<.001)$, suggesting that older individuals were somewhat likely to be closer to death (i.e., higher distance-to-death scores as "less negative," or closer to zero being associated with higher ages). In sum, the data structure suggests only partial overlap between the two time dimensions, and model inferences are most relevant for the 70 to 90 age span and/or the decade prior to death.

\section{Results}

\section{Comparing Age-Related and Mortality-Related Changes in Life Satisfaction}

Before examining intraindividual changes in life satisfaction, we checked the relative amount of between-person and within-person variance in the data. The intraclass correlation was .48 (as com- 
puted using a random intercept-only model). In other words, $48 \%$ of the total variation in life satisfaction was between-person variance, with the remainder (52\%) being within-person variation. The data thus appeared to contain both substantial amounts of betweenperson differences and within-person variation over time. Noting that there was indeed intraindividual variation to model, four growth models, 2 types of change (linear and multiphase) $\times 2$ time metrics (age and distance-to-death) were used to describe and evaluate how the noted changes in life satisfaction were structured over time.

Linear models. Parameter estimates and fit statistics for the linear models, with either age or distance to death as the time metric, are presented in Table 2. The distance-to-death model provided a better fit to the data, as evaluated by relative overall model fit criteria (Akaike information criterion [AIC] $=80,008$ for the age model; AIC $=79,645$ for the distance-to-death model; lower AIC indicates better relative model fit). Relative fit was also evaluated with regard to the additional amount of explained variance, formally conceptualized as the proportional reduction of

Table 1

Descriptive Statistics for Life Satisfaction Over Age at Assessment and Distance to Death (DtD)

\begin{tabular}{|c|c|c|c|c|c|c|c|}
\hline \multirow[b]{2}{*}{$\operatorname{Age}^{\mathrm{a}}$} & \multicolumn{3}{|c|}{ Chronological age } & \multicolumn{4}{|c|}{ Distance to death (DtD) } \\
\hline & $n$ & $M$ & $S D$ & $\mathrm{DtD}^{\mathrm{a}}$ & $n$ & $M$ & $S D$ \\
\hline 70 & 634 & 48.05 & 15.05 & 22 & 4 & 59.55 & 11.17 \\
\hline 71 & 660 & 48.72 & 14.57 & 21 & 10 & 49.87 & 13.26 \\
\hline 72 & 663 & 48.85 & 14.28 & 20 & 18 & 50.23 & 9.76 \\
\hline 73 & 666 & 47.99 & 14.91 & 19 & 26 & 53.10 & 12.54 \\
\hline 74 & 647 & 48.47 & 14.34 & 18 & 37 & 49.70 & 14.94 \\
\hline 75 & 642 & 47.88 & 14.94 & 17 & 54 & 53.10 & 12.58 \\
\hline 76 & 636 & 49.12 & 14.98 & 16 & 79 & 52.81 & 12.89 \\
\hline 77 & 629 & 48.55 & 14.61 & 15 & 119 & 53.02 & 12.25 \\
\hline 78 & 606 & 46.65 & 14.97 & 14 & 157 & 53.82 & 11.31 \\
\hline 79 & 552 & 48.18 & 15.33 & 13 & 207 & 54.02 & 11.85 \\
\hline 80 & 520 & 47.48 & 14.82 & 12 & 263 & 52.42 & 12.38 \\
\hline 81 & 474 & 46.77 & 15.65 & 11 & 341 & 52.80 & 12.76 \\
\hline 82 & 444 & 47.60 & 14.76 & 10 & 402 & 51.30 & 13.57 \\
\hline 83 & 402 & 47.53 & 14.80 & 9 & 484 & 50.86 & 13.68 \\
\hline 84 & 360 & 47.29 & 14.33 & 8 & 567 & 49.27 & 14.15 \\
\hline 85 & 316 & 46.83 & 13.93 & 7 & 664 & 49.83 & 14.11 \\
\hline 86 & 276 & 45.50 & 15.90 & 6 & 789 & 48.87 & 13.80 \\
\hline 87 & 236 & 46.10 & 15.89 & 5 & 940 & 48.40 & 14.23 \\
\hline 88 & 200 & 45.00 & 15.53 & 4 & 1,101 & 47.24 & 14.83 \\
\hline 89 & 152 & 46.69 & 13.47 & 3 & 1,229 & 46.30 & 14.78 \\
\hline 90 & 129 & 45.42 & 15.57 & 2 & 1,365 & 45.30 & 15.45 \\
\hline 91 & 92 & 46.29 & 16.41 & 1 & 1,303 & 41.14 & 16.62 \\
\hline 92 & 68 & 47.69 & 13.90 & 0 & 3 & 43.42 & 12.90 \\
\hline 93 & 57 & 44.10 & 16.30 & & & & \\
\hline 94 & 36 & 43.96 & 16.13 & & & & \\
\hline 95 & 25 & 47.55 & 18.33 & & & & \\
\hline 96 & 19 & 46.48 & 19.64 & & & & \\
\hline 97 & 8 & 34.55 & 18.87 & & & & \\
\hline 98 & 8 & 35.35 & 15.33 & & & & \\
\hline 99 & 4 & 32.13 & 23.19 & & & & \\
\hline 100 & 1 & 56.32 & - & & & & \\
\hline
\end{tabular}

Note. $\quad N=1,637$ who provided 10,162 observations. Scores standardized to a $T$ metric $(M=50 ; S D=10)$ using the entire Socio-Economic Panel Study longitudinal sample as the reference frame $(M=7.02, S D=1.55$; see Lucas, Clark, Georgellis, \& Diener, 2003). The dash indicates that only one observation was available at age 100 , which precludes computing the standard deviation.

${ }^{\text {a }}$ In years.
Table 2

Linear Growth Models for Life Satisfaction Over Chronological Age and Distance to Death

\begin{tabular}{|c|c|c|c|c|}
\hline \multirow[b]{3}{*}{ Parameter } & \multicolumn{4}{|c|}{ Life satisfaction } \\
\hline & \multicolumn{2}{|c|}{ Chronological age } & \multicolumn{2}{|c|}{ Distance to death } \\
\hline & Estimate & $S E$ & Estimate & $S E$ \\
\hline \multicolumn{5}{|l|}{ Fixed effects estimates } \\
\hline Intercept, $a_{O O}{ }^{\mathrm{a}}$ & $42.10^{*}$ & 0.45 & $42.30^{*}$ & 0.39 \\
\hline Slope, $a_{O I}{ }^{\mathrm{b}}$ & $-0.63^{*}$ & 0.04 & $-1.02^{*}$ & 0.05 \\
\hline \multicolumn{5}{|l|}{ Random effects estimates } \\
\hline Variance intercept & $159.78^{*}$ & 10.82 & $169.76^{*}$ & 8.67 \\
\hline Variance slope & $0.65^{*}$ & 0.08 & $0.93^{*}$ & 0.11 \\
\hline Cov. intercept, slope & $5.21^{*}$ & 0.80 & $8.85^{*}$ & 0.90 \\
\hline Residual variance & $107.51^{*}$ & 1.74 & $104.95^{*}$ & 1.70 \\
\hline Number parameters & \multicolumn{2}{|c|}{6} & \multicolumn{2}{|c|}{6} \\
\hline$-2 \mathrm{LL}$ & \multirow{2}{*}{\multicolumn{2}{|c|}{$\begin{array}{l}79,996 \\
80,008\end{array}$}} & \multicolumn{2}{|c|}{79,633} \\
\hline AIC & & & \multicolumn{2}{|c|}{79,645} \\
\hline
\end{tabular}

Note. Unstandardized estimates and standard errors are presented. $N=$ 1,637 who provided 10,162 observations. Scores standardized to a $T$ metric $(M=50 ; S D=10)$ using the entire Socio-Economic Panel Study longitudinal sample as the reference frame $(M=7.02, S D=1.55$; see Lucas et al., 2003). Cov. = covariance; AIC $=$ Akaike information criterion; $-2 \mathrm{LL}=-2 \log$ likelihood, relative model fit statistics.

antercept is centered at age 85 for age-based model, or at death for distance-to-death model. ${ }^{\text {bSlope }}$ or rate of change is scaled in $T$ units per year.

"p $<.05$ or below.

prediction error (i.e., change in pseudo- $R^{2}$ ), when either age or distance to death was added to the within-person (Level 1) portion of the model (Snijders \& Bosker, 1999). ${ }^{2}$ The change in pseudo- $R^{2}$ was .145 for the distance-to-death metric as compared to .125 for the age metric. Taken together, the assessments of relative overall model fit and proportion of explained variance both suggest that distance to death provides a better fitting and more efficient description of longitudinal changes in life satisfaction aspects of the data than does chronological age. We also note that, on average, the age model shows significant age-associated decline (-0.63 $T$-score units per year), whereas the distance-to-death model shows a relatively more pronounced mortality-associated decline (-1.02 $T$-score units per year).

Multiphase models. Subsequently, models incorporating multiple phases of change (i.e., Equation 2) over both age and distance to death were examined. Our intent was to empirically evaluate whether multiphase models of change were better than singlephase models of change. Parameter estimates and model fit indices

\footnotetext{
${ }^{2}$ Following the general framework outlined by Snijders and Bosker (1999, pp. 99-105), we calculated the explained proportion of withinperson variance as

$$
\Delta \text { pseudo- } R^{2}=1-\left(\sigma_{\mathrm{e}(\mathrm{c})}^{2} / \sigma_{\mathrm{e}(\mathrm{u})}^{2}\right),
$$

where $\sigma_{\mathrm{e}(\mathrm{u})}^{2}$ is the residual within-person variance obtained from an unconditional or intercept-only model (for our data $=122.81$ ) and $\sigma_{\mathrm{e}(\mathrm{c})}^{2}$ is the parallel term from the conditional model that includes the time variable, age or distance to death (for our data $=107.51$ and 104.95, respectively). It was also noted that the inclusion of both time metrics only marginally increased the explained variance further $\left(\Delta\right.$ pseudo- $\left.R^{2}=.147\right)$.
} 
are reported in Table 3. The multiple-phase models provided better fit to the data for both age $(\mathrm{AIC}=79,917)$ and distance-to-death $(\mathrm{AIC}=79,445)$ time metrics than the linear counterparts $(\mathrm{AIC}=$ 80,008 and $\mathrm{AIC}=79,645$, respectively). Over chronological age, prototypical change was characterized by a decline of $-0.47 \mathrm{~T}$ units per year until 81.23 years of age $(S E=0.39)$, where the rate of decline accelerated to $-0.56 T$ units per year. These parameters suggest that normative age-related intraindividual decline in life satisfaction may be somewhat more pronounced in very old age (i.e., 85+ years of age; see Baltes \& Smith, 2003) than in old age (i.e., 70 to 85 years of age). Over distance to death, prototypical multiphase changes in life satisfaction (of the terminal decline type) were characterized by a preterminal decline of $-0.64 T$ units per year and a transition at 4.19 years $(S E=0.17)$ prior to death to steeper terminal decline of $-1.94 T$ units per year. Further, as was the case for the linear models, comparison across time metrics revealed that the distance to death time metric provided for better and more efficient representation of the intraindividual changes in life satisfaction and the interindividual differences therein (AIC $=$ 79,917 for age vs. AIC $=79,445$ for distance to death) ${ }^{3}$

Overall, the model closest to the terminal decline hypothesisone that represented late-life changes in life satisfaction over a distance-to-death time metric as a transition between two phases, with the latter phase characterized by steeper decline than the former-provided the best fit (of the models tested) to the data. Prototypical and model-implied intraindividual changes in life satisfaction for a random selection of 100 participants are shown in

Table 3

Multiphase Growth Models for Life Satisfaction Over Chronological Age and Distance to Death

\begin{tabular}{|c|c|c|c|c|}
\hline \multirow[b]{3}{*}{ Parameter } & \multicolumn{4}{|c|}{ Life satisfaction } \\
\hline & \multicolumn{2}{|c|}{ Chronological age } & \multicolumn{2}{|c|}{ Distance to death } \\
\hline & Estimate & $S E$ & Estimate & $S E$ \\
\hline \multicolumn{5}{|l|}{ Fixed effects estimates } \\
\hline Intercept, $a_{O O}{ }^{\mathrm{a}}$ & $44.67^{*}$ & 0.53 & $48.14^{*}$ & 0.42 \\
\hline Change point, $k$ & $81.23^{*}$ & 0.39 & $4.19^{*}$ & 0.17 \\
\hline Slope $1, a_{O O}^{\mathrm{b}}$ & $-0.47^{*}$ & 0.11 & $-0.64^{*}$ & 0.06 \\
\hline Slope $2, a_{O O}{ }^{\mathrm{b}}$ & $-0.56^{*}$ & 0.06 & $-1.94^{*}$ & 0.15 \\
\hline \multicolumn{5}{|l|}{ Random effects estimates } \\
\hline Variance intercept & $181.23^{*}$ & 12.08 & $128.60^{*}$ & 7.02 \\
\hline Variance slope 1 & $2.11^{*}$ & 0.43 & $0.78^{*}$ & 0.14 \\
\hline Variance slope 2 & $1.38^{*}$ & 0.18 & $9.11^{*}$ & 1.20 \\
\hline Cov. intercept, slope 1 & $-10.67^{*}$ & 2.08 & $6.58^{*}$ & 0.88 \\
\hline Cov. intercept, slope 2 & $9.45^{*}$ & 1.25 & $-7.01^{*}$ & 2.04 \\
\hline Cov. slope 1 , slope 2 & $-0.61^{*}$ & 0.26 & -0.16 & 0.32 \\
\hline Residual variance & $103.98^{*}$ & 1.71 & $97.49^{*}$ & 1.66 \\
\hline Number parameters & \multicolumn{2}{|c|}{11} & \multicolumn{2}{|c|}{11} \\
\hline$-2 \mathrm{LL}$ & \multicolumn{2}{|c|}{79,895} & \multicolumn{2}{|c|}{79,423} \\
\hline AIC & \multicolumn{2}{|c|}{79,917} & \multicolumn{2}{|c|}{79,445} \\
\hline
\end{tabular}

Note. Unstandardized estimates and standard errors are presented. $N=$ 1,637 who provided 10,162 observations. Scores standardized to a $T$ metric $(M=50, S D=10)$ using the entire Socio-Economic Panel Study longitudinal sample as the reference frame $(M=7.02, S D=1.55$; see Lucas et al., 2003). Cov. = covariance; AIC $=$ Akaike information criterion; $-2 \mathrm{LL}=-2 \log$ likelihood, relative model fit statistics.

${ }^{a}$ Intercept is centered at the change point in both models. ${ }^{\mathrm{b}}$ Slope or rate of change in $T$ units per year.

${ }^{*} p<.05$ or below.
Figure 1. Prototypically, the rate of life satisfaction decline steepened by a factor of three at around 4 years prior to death. In addition to these fixed effects or "prototypical changes," the multiphase distance-to-death model reported in Table 3 also revealed interindividual differences in preterminal phase slopes, terminal phase slopes, and the level of life satisfaction at the point of transition. The pattern of covariances reflects, as would be expected, that individuals who exhibited steeper preterminal declines tended to arrive at the transition phase at relatively lower levels of life satisfaction $\left(\sigma_{u O u l}=6.58\right.$ or in correlation units $\left.r_{u 0 u l}=.66\right)$. Further, individuals who arrived at low levels were somewhat more likely to exhibit shallower decline in the terminal phase $\left(r_{\text {uou } 2}=-.20\right)$. Rates of decline in the preterminal phase, however, were not significantly correlated with rates of decline in the terminal phase $\left(r_{u 1 u 2}=-.06\right)$.

\section{Interindividual Differences in Terminal Decline}

To push the articulation of terminal decline from the notion of a population-level transition parameter toward individual-level transitions, we also explored possible interindividual differences in the location of the change point. To do so, we used data from a subsample of individuals who provided extensive longitudinal data and fitted random effects change-point models. As outlined above, the model captured interindividual differences in the point of transition between preterminal and terminal phases of decline by including $k_{i}$ as a random effect (Cudeck \& Harring, 2007; Cudeck \& Klebe, 2002).

Subsample. The increase in model complexity required fitting only those individuals who had provided $\sim 12+$ observations ( $n=$ 400 ; $54 \%$ women; age at death, $M=81.60$ years, $S D=6.66$; range: 71-101). This subsample did not differ from our larger sample of decedents on demographic characteristics of gender, years of education, and age at death, $F \mathrm{~s}(1,1636)<1.10$, all $p \mathrm{~s}>$ .10. Further, as reported in Columns 1 and 2 of Table 4, the normative pattern of change for these 400 persons was very similar to that found in the total sample (e.g., location of the change point at 4.53 years vs. the 4.19 years reported in Table 3 ). In short, the subsample appears to exhibit the same multiphase terminal decline phenomena found in the total sample.

Interindividual differences in onset of terminal phase. The right panel of Table 4 shows that a model relaxing the strict assumption that individuals all transitioned into the terminal phase at exactly the same time fit our data better than the fixed-changepoint model (deviance information criterion [DIC] $=30,383$ vs

\footnotetext{
${ }^{3}$ Consistent with our finding of increased steepness of decline prior to death, a model specifying linear and quadratic change in life satisfaction over distance-to-death was found to fit our data better $(-2 \mathrm{LL}=79,470)$ than a model with linear change only $(-2 \mathrm{LL}=79,633 ; \Delta-2 \mathrm{LL}=163)$ However, the two-phase model with a change point 4.19 years prior to death still provided better relative model fit $(\mathrm{AIC}=79,445)$ than the quadratic model $(\mathrm{AIC}=79,490 ; \Delta \mathrm{AIC}=45)$. We also explored quadratic trends over chronological age, but these were not significantly different from zero (neither with nor without random effects for the quadratic component).
} 


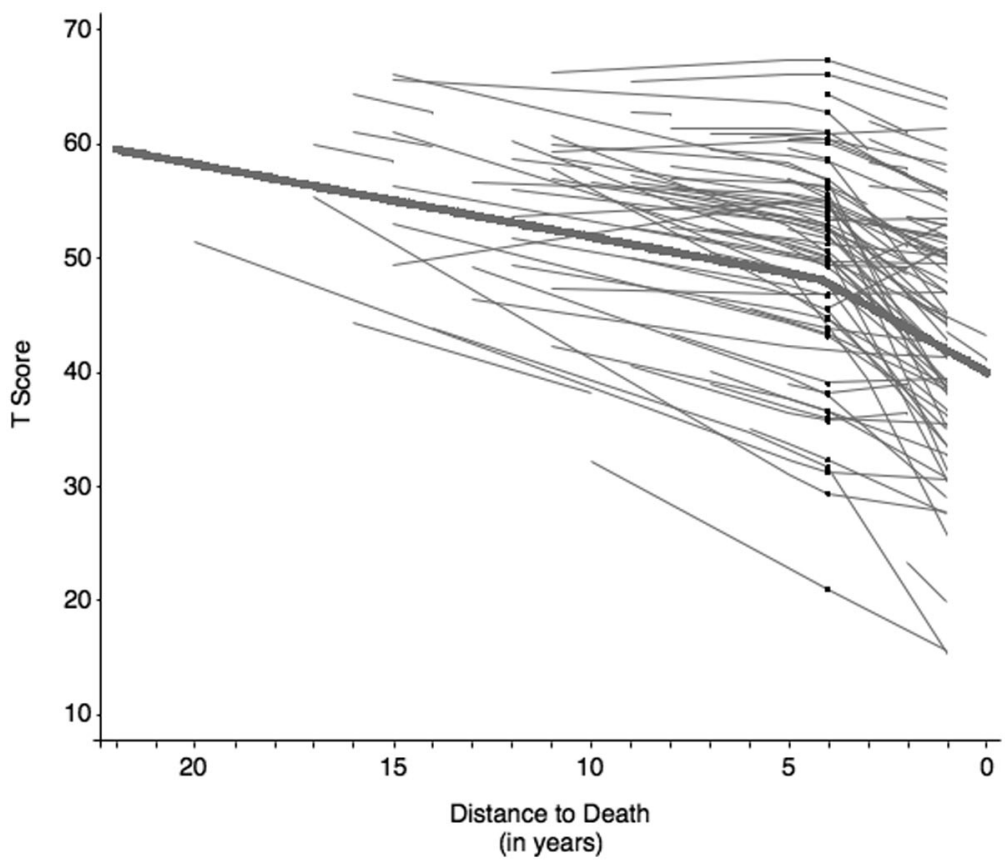

Figure 1. Estimates from the optimal multiphase growth model over distance to death in life satisfaction, as identified using 22-wave yearly longitudinal data from now-deceased 70- to 100-year-old Socio-Economic Panel Study participants $(N=1,637$; see columns 3 and 4 of Table 3). Prototypical (thick line) and model-implied intraindividual changes in life satisfaction for a random selection of 100 deceased participants (thin lines) are shown. At a change point 4.19 years prior to death, the rate of decline steepened from the preterminal phase $(-0.64 T$-score units per year) to the terminal phase $(-1.94 T$-score units per year) by a factor of three.

DIC $=30,453) .{ }^{4}$ Table 4 also reveals that the normative pattern of change, with relatively shallow preterminal decline, a transition at around 4 years prior to death, and relatively steep terminal decline, follows the same general form as before. Most importantly, we found notable interindividual differences in the location of the change point $\left(\sigma_{\mathrm{k}}^{2}=12.08\right)$, indicating that individuals transition from one phase to another at different times. Illustrating this finding, Figure 2 shows prototypical and model-implied intraindividual changes in life satisfaction for a random selection of 100 participants from the subsample. Although on average this subset of individuals transitioned to the terminal phase at 3.67 years before death, some individuals entered earlier (e.g., 6 years prior to death), some later (e.g., 1 year prior), and some not at all (i.e., at a point projected to be after their actual death). More specifically, the estimated parameters transition points spanning the range from 13 years prior to death to 1 year after death. Although this may seem somewhat strange, consider that, theoretically, some individuals die before they enter terminal decline (e.g., dying from an acute disease or event like an accident). In the $n=400$ subsample used here, individual change points were estimated to be at or after death for 28 individuals. Follow-up examinations of these individuals indicated that their data series were each better characterized by single-phase linear declines rather than multiphases-a further indication that they did not ever enter a terminal-decline phase. Finally, as would be expected, the pattern of covariances suggests that individuals who exhibited steeper preterminal decline transitioned to the terminal phase of decline somewhat earlier $\left(r_{u l u 3}=\right.$ $.55)$.
Predictors of individual onset. In a final step, we explored how differences in the timing of transition to the terminal-decline phase might relate to interindividual difference characteristics. To do so, the location of the change point, $k_{i}$, was regressed on age at death, sex, and education, and their two-way and three-way interactions. ${ }^{5}$ In these analyses, presented in Table 5, only $2 \%$ of the variance in the location of the change point was accounted for. We did not find evidence of statistically significant differences across sex or levels of education, although nominally there was an indication that being a woman, as opposed to a man, was associated with a longer terminal phase and steeper terminal decline. There was, however, a significant relationship between age at death and the location of the change point $\left(a_{13}=0.06\right)$, with older age at death associated with having spent more time in the terminal decline phase. This effect amounted to some 22 more days in the terminal phase per additional year lived $(0.06 \times 365$ days $)$.

\footnotetext{
${ }^{4}$ DIC $=$ Deviance information criteria, a measure of relative model fit, intended as a generalization of the AIC. Having used Gibbs sampling estimation to conduct the analysis, we report the DIC generalization in lieu of the AIC. Interpretation is the same, with lower values indicating a better relative model fit.

${ }^{5}$ We also used age at death, sex, and education as well as their interaction terms as predictors of level, preterminal slope, and terminal slope. None of the covariates, however, were significantly related to these model parameters.
} 
Table 4

Multiphase Growth Models of Life Satisfaction Over Distance to Death With Change Points as Fixed or Random

\begin{tabular}{|c|c|c|c|c|}
\hline \multirow[b]{3}{*}{ Parameter } & \multicolumn{4}{|c|}{ Life satisfaction } \\
\hline & \multicolumn{2}{|c|}{$\begin{array}{l}\text { Fixed-change } \\
\text { point }\end{array}$} & \multicolumn{2}{|c|}{$\begin{array}{l}\text { Random-change } \\
\text { point }\end{array}$} \\
\hline & Estimate & $S E$ & Estimate & $S E$ \\
\hline \multicolumn{5}{|l|}{ Fixed effects estimates } \\
\hline Intercept, $a_{O O}^{\mathrm{a}}$ & $47.52 *$ & 0.75 & $48.65^{*}$ & 0.78 \\
\hline Change point, $k$ or $a_{O 3}$ & $4.53^{*}$ & 0.38 & $3.67^{*}$ & 0.69 \\
\hline Slope $1, a_{O O}^{\mathrm{b}}$ & $-0.56^{*}$ & 0.08 & $-0.28^{*}$ & 0.10 \\
\hline Slope $2, a_{O O}^{\mathrm{b}}$ & $-2.17^{*}$ & 0.27 & $-3.51^{*}$ & 0.83 \\
\hline \multicolumn{5}{|l|}{ Random effects estimates } \\
\hline Variance intercept & $128.90^{*}$ & 11.95 & $84.85^{*}$ & 10.70 \\
\hline Variance change point & \multicolumn{2}{|c|}{ n.e. } & $12.08^{*}$ & 2.95 \\
\hline Variance slope 1 & $0.60^{*}$ & 0.13 & $0.26^{*}$ & 0.12 \\
\hline Variance slope 2 & $6.40^{*}$ & 1.75 & $5.28^{*}$ & 1.74 \\
\hline $\begin{array}{l}\text { Cov. intercept, change } \\
\text { point }\end{array}$ & \multicolumn{2}{|c|}{ n.e. } & $18.66^{*}$ & 4.06 \\
\hline Cov. intercept, slope 1 & $6.13^{*}$ & 1.11 & $3.13^{*}$ & 0.87 \\
\hline Cov. intercept, slope 2 & -5.47 & 2.87 & $-18.54^{*}$ & 4.06 \\
\hline $\begin{array}{l}\text { Cov. Change point, } \\
\text { slope } 1\end{array}$ & \multicolumn{2}{|c|}{ n.e. } & $0.98^{*}$ & 0.41 \\
\hline $\begin{array}{l}\text { Cov. Change point, } \\
\text { slope } 2\end{array}$ & \multicolumn{2}{|c|}{ n.e. } & -2.45 & 2.12 \\
\hline Cov. slope 1 , slope 2 & 0.03 & 0.32 & $-0.70^{*}$ & 0.22 \\
\hline Residual variance & $87.96^{*}$ & 2.27 & $87.86^{*}$ & 2.66 \\
\hline Number parameters & \multirow{2}{*}{\multicolumn{2}{|c|}{$\begin{array}{c}11 \\
30,453\end{array}$}} & \multicolumn{2}{|c|}{15} \\
\hline $\mathrm{DIC}$ & & & \multicolumn{2}{|c|}{30,383} \\
\hline
\end{tabular}

Note. Unstandardized estimates and standard errors are presented. $N=$ 400. Scores standardized to a $T$ metric $(M=50 ; S D=10)$ using the entire Socio-Economic Panel Study longitudinal sample as the reference frame $(M=7.02, S D=1.55$; see Lucas et al., 2003). Cov. = covariance; n.e. $=$ not estimated (i.e., assumed to be 0.0); DIC $=$ deviance information criterion, relative model fit statistic.

${ }^{a}$ Intercept is centered at the change point in both models. ${ }^{\mathrm{b}}$ Slope or rate of change in $T$ units per year.

$* p<.05$ or below.

\section{Discussion}

The objective of this study was to identify and describe terminal decline in life satisfaction in old age. Using 22-wave longitudinal data from now deceased 70 - to 100-year-old participants in the SOEP study, we found that individual differences in late-life intraindividual changes in life satisfaction were better described using a distance-to-death rather than a distance-from-birth time metric. Of the four growth curve models tested, a model that articulated notions of terminal decline by incorporating two phases of change over distance to death provided the best fit to the data. This model revealed a transition point about 4 years prior to death at which the prototypical rate of decline steepened by a factor of three from the preterminal phase to the terminal phase. Follow-up analyses indicated that men and women and individuals of differing educational levels experienced, on average, comparable late-life declines in life satisfaction, but individuals who died at later ages tended to spend more time in terminal periods of decline.

\section{Age-Related and Mortality-Related Changes in Life Satisfaction}

Although data vary somewhat across cultures and subgroups, individuals for the most part report being happy or satisfied with their lives (Diener \& Diener, 1996). For example, Lucas et al. (2003) found that the vast majority ( $88 \%$ ) of the $\sim 22,000$ participants in the larger SOEP sample reported life satisfaction scores above neutral (i.e., $>5$ on the 0 to 10 scale). Similarly, within the older and now deceased segment of the same sample used here, $85 \%$ of reports $(8,669$ of 10,162 observations) were at or above neutral. When these 70 - to 100 -year-olds were broken down by age and distance to death, however, we found systematic declines in life satisfaction such that average levels were below the neutral point at ages 97 to 99 (see Table 1) and at death for individuals who died older than age 85 (i.e., intercept $=4.97$ in follow-up analyses applying the multiphase model to data in the original scale units). Thus, in this nationally representative sample from a highly developed country a number of individuals in very old age and/or the last few years of life report being fairly unsatisfied. Without delving into the moral and ethical issues of whether society should knowingly tolerate decline below neutral levels of satisfaction (see Baltes, 2006), we simply observe, in the context of aging and longevity, that there appears to be a "soon to die" segment of the population that is not satisfied. Whether or not such feelings can be alleviated should be examined further.

When examining how late-life longitudinal changes in life satisfaction were structured, we found, somewhat in contrast with other reports, indications of sizeable age-related decline (e.g., Charles, Reynolds, \& Gatz, 2001; Mroczek \& Kolarz, 1998). The extent of decline in the present study (e.g., $-0.63 T$-score units per year in the linear age-based model) was somewhat steeper than that found (or implied by cross-sectional age differences) in several previous studies (e.g., no age differences across the lifespan in Lucas \& Gohm, 2000). This may have been because we only selected older participants (older than in most other studies) who, in addition, were known to have died. To explore this possibility, we did follow-up analyses on all SOEP participants who provided data after age 70, independent of their mortality status $(N=$ $3,519)$, which revealed a somewhat shallower age gradient $(-0.54$ $T$ units per year). In other words, there is some evidence that the mortality-based selection criterion employed in this study contributed to the steepness of age-related decline in life satisfaction found here, as compared to that found in other studies. ${ }^{6}$

Our finding that distance-to-death models fit the data relatively better than age-based models speaks to the utility of examining mortality selection processes explicitly. Organizing the change gradients according to the selection criterion (i.e., death) revealed that progressive processes leading toward death may also be those that drive changes in life satisfaction occurring in old age. In other words, mortality-related processes may be a major underlying influence when late-life changes in well-being are indeed observed. Parallel to evidence accumulating for various measures of

\footnotetext{
${ }^{6}$ When we further included only participants who had not died by the year 2005, we found an even shallower age gradient of $-0.43(S E=0.03)$, $p<.001$.
} 


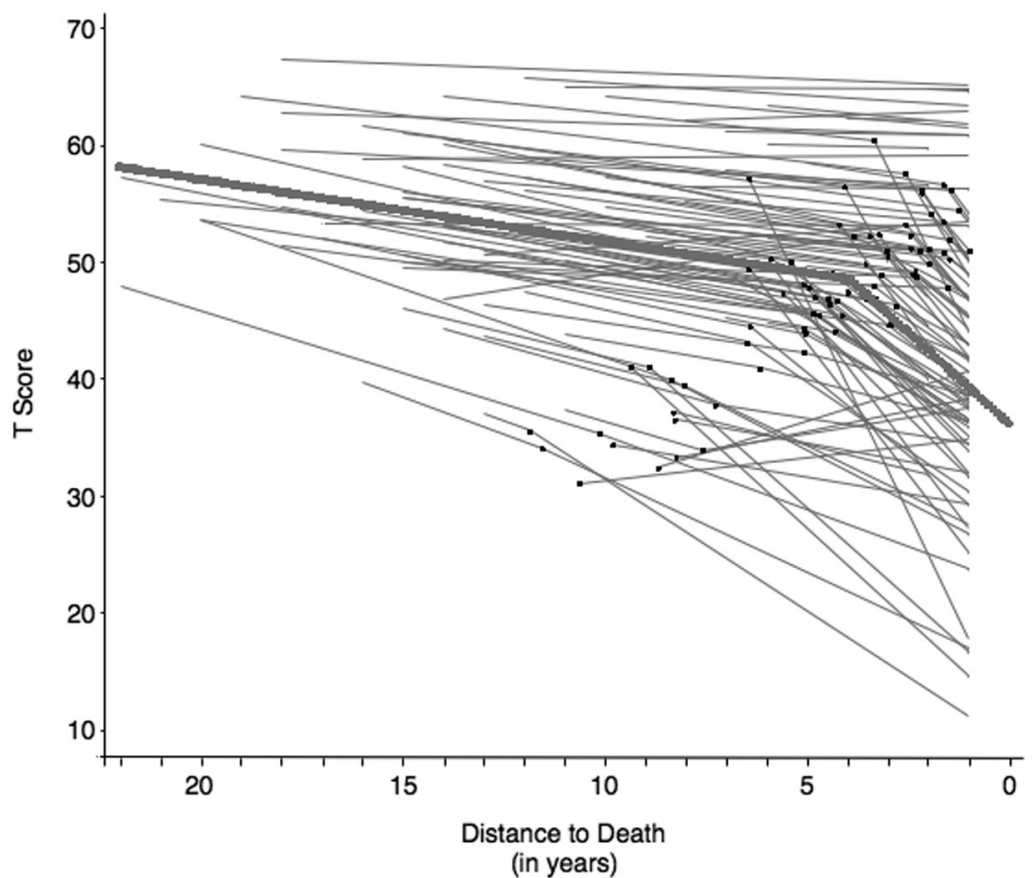

Figure 2. Estimates from the optimal multiphase growth model over distance-to-death in life satisfaction with interindividual differences in the change point, as identified in a subset of Socio-Economic Panel Study participants who provided a large number of longitudinal observations ( $\sim 12+$ observations; $n=400$; see columns 3 and 4 of Table 4). Prototypical (thick line) and model-implied intraindividual changes in life satisfaction for a random selection of 100 deceased participants (thin lines) are shown. Large interindividual differences in the location of the change point to more pronounced late-life decline in life satisfaction can be seen. Although on average this subset of individuals transitioned to the terminal phase at 3.67 years before death, some individuals entered earlier (e.g., 6 years prior to death), some later (e.g., 1 year prior), and some hardly or not at all.

cognitive functioning (Bäckman \& MacDonald, 2006) and recent reports based on multi-item questionnaires of life satisfaction from the Normative Aging Study (Mroczek \& Spiro, 2005) and the Berlin Aging Study (Gerstorf et al., 2008), the present findings from the nationally representative SOEP suggest that

Table 5

Multiple Regression of Interindividual Differences in the Location of the Change Point on Demographic Characteristics

\begin{tabular}{lcc}
\hline & \multicolumn{2}{c}{ Change point } \\
\cline { 2 - 3 } \multicolumn{1}{c}{ Predictor } & $B$ & $S E$ \\
\hline Intercept $a_{03}$ & $3.68^{*}$ & 0.251 \\
Age at death $a_{13}$ & $0.06^{*}$ & 0.029 \\
Sex $a_{23}$ & 0.48 & 0.334 \\
Education $a_{33}$ & 0.02 & 0.160 \\
Age at Death $\times$ Sex $a_{43}$ & -0.08 & 0.042 \\
Age at Death $\times$ Education $a_{53}$ & -0.01 & 0.017 \\
Sex $\times$ Education $a_{63}$ & -0.06 & 0.205 \\
Age $\times$ Sex $\times$ Education $a_{73}$ & -0.00 & 0.023 \\
\hline
\end{tabular}

Note. Sex: $0=$ men, $1=$ women. $N=400$. The predictive effect of age at death also held when level, preterminal slope, and terminal slope were used as additional predictors for the location of the change point. Excluding participants with individual change points estimated to be at or after death $(n=28)$ revealed substantively the same results.

${ }^{*} p<.05$ or below. within-person changes in well-being occurring late in life can also be structured as a mortality-related process. Impending death appears to be a progressive process that encompasses numerous domains.

\section{Terminal Decline in Life Satisfaction: Normative Trends and Interindividual Differences}

Following the terminal decline hypothesis (e.g., Kleemeier, 1962), multiphase growth models were used to identify when individuals transition from a preterminal phase of normative gradual decline to a terminal phase of pronounced decline. Estimated to be some 4 years before death, the placement of this transition is consistent with previous reports for both the cognitive domain ( 8 years, Sliwinski et al., 2006; 4 years, Wilson et al., 2003) and the well-being domain (4 years, Gerstorf et al., 2008). Such comparability with previous reports is noteworthy given the constraint of having available mortality information on a yearly basis only (e.g., for participants whose final interview occurred in the calendar year prior to death, the recorded lag could range from a minimum of 1 day to a maximum of just short of 1.5 years). We also note consistencies with these earlier reports in that average terminalphase decline amounted to $-1.94 T$ units per year (earlier reports correspond to effects ranging between -0.8 and -2.2 ), three times the decline noted for the preterminal phase (earlier reports found 
terminal-phase decline to be between 2 to 12 times as steep). ${ }^{7}$ Together, the transition point and rate of terminal decline suggest that the prototypical individual's life satisfaction declines nearly a full standard deviation over the last 4 years of life (from 48 to 40 on our $T$-unit scale or from 7 to 5.5 on the raw-unit scale). We highlight, however, that all these reports (including our own) depict average population level estimates and make an assumption that the location of the transition point is invariant across individuals.

To address this concern, we initiated an exploration into how the timing of the onset of terminal decline may differ across individuals using a random change-point multiphase growth model where the restrictive assumption noted above was relaxed. Our initial evidence suggests that people do appear to enter the terminaldecline phase at different times, ranging from 13 years prior to death to just prior to death or even not at all. We find this model to be consistent with the idea that although terminal decline is normative, some individuals will not experience such transitions because they die, presumably of some other cause (e.g., accident), before they entered terminal decline. Persons following such a trajectory do appear in the data, treating time (e.g., distance to death) as a continuous variable and assuming a normal distribution of interindividual differences in the onset allowed for this theoretically consistent possibility.

In a subsequent step, we explored how the noted differences in onset of terminal decline related to differences in age at death, sex, and education. Onset was not related to sex and education in a statistically significant manner. However, differences in onset were related to age of death, in that individuals dying at later ages appear to have spent more time in the terminal-decline phase (about 7 months per additional decade lived). Before interpreting these findings, we note explicitly that the absence or presence of significant effects from this post hoc regression of estimated parameters on a small set of interindividual difference variables suffers from limitations in the data (e.g., yearly assessments, missing data). Articulate and precise tests of interindividual difference hypotheses would, at the very least, require an increased density of observations. Strong conclusions from this primarily exploratory post hoc analysis are not warranted.

With that duly noted, our cautious interpretation, perhaps still a speculation, is that it is not age per se that matters (for life satisfaction) but rather a combination of closeness to death and the age at which this closeness appears. Individual-based notions of distinguishing multiple phases in old age, for example, highlight that transitions from the "Third Age" to the "Fourth Age" are primarily linked to the lifespan of a given individual and can thus occur at very different ages (e.g., around age 60 for some or around age 90 for others; see Figure 1 in Baltes \& Labouvie, 1973, reprinted in Hertzog \& Nesselroade, 2003; Baltes \& Smith, 2003). Our finding that the years prior to death may be more dysfunctional in older ages (e.g., drawn-out decline; see also Crimmins, 2001 ) is consistent with the idea that terminal decline in well-being may, in part, be a consequence of age-related overburden. It may be that individuals who survive into very old age are at the limits of their adaptive capacity (Smith \& Gerstorf, 2004). As other systems begin to break down, an already compromised well-being regulation system becomes overwhelmed and exhibits precipitous decline. So, whereas individuals in the "Third Age" (e.g., 70-84 years) may still have the resources to ward off the detrimental effects of impending mortality, those in the "Fourth Age" (85+ years) may not. An alternative, perhaps more contentious possibility is that declines in well-being are an indication that the well-being regulation mechanism falls apart (e.g., the mechanics of self-regulation become more and more compromised in processing rewards) and that this increasing dysfunction itself contributes to the dying process. Speculating from evidence regarding the age sensitivity of corticostriatal connections and dopamine-dependent processes implicated in reward processing (e.g., Gerhardt, Cass, Yi, Zhang, \& Gash, 2002), it may be that as individuals are no longer able to process rewards, life is no longer rewarding and the desire to live decreases.

This initial analysis of interindividual differences in terminal decline was in some ways a theoretically informed exploratory analysis, driven in large part by the limitations of the data (e.g., relatively small number of variables, lack of information about cognitive functioning or cause of death), and is unable to address the above speculations. Although limited, we hope that it does provide an initial look at and generate some discussion about how theoretical propositions regarding interindividual differences in terminal decline may be articulated, modeled, and examined.

\section{Limitations and Outlook}

Ideally, the statistical methods used in an analysis should be precise articulations of the theoretical models one seeks to test. In this article, we have sought to apply and extend models that correspond to theoretical notions of how terminal decline may manifest in late-life changes in life satisfaction. In an attempt to do so, we began by modeling linear changes over an age time metric, replaced the time metric with distance-to-death as a more direct proxy of the mortality-related processes implicated in terminal decline and allowed for explicit transition between preterminal and terminal phases of decline. Finally, we articulated a more individualized version of terminal decline using a multiphase random change-point model wherein individuals differ in timing of onset of terminal decline. Proceeding in this manner, we ended up at a model that represents (and requires) some of the latest innovations in statistical estimation and remains at the edge of what is possible with currently available software (see Cudeck \& Harring, 2007; Wang \& McArdle, 2008). Although we are sure that such developments allow for more precise articulation of the theory (e.g., individual differences in onset of terminal decline), we still interpret the results with some caution. The initial descriptions provided here, though, suggest that further examinations with more advanced tools and intensive predeath repeated measures should be pursued. At the same time, these initial results obtained with the advanced modeling tools suggest that the theory can be articulated and (with sufficient data) tested in detail. In turn, this may require some further refinement of the theory itself. Specific hypotheses to be generated include specification of when the onset of terminal

\footnotetext{
${ }^{7}$ Comparing standardized units across studies is, of course, open to debate given that standardizations depend upon sample characteristics. However, we opted for this approach in a first attempt to compare rates of terminal decline across studies and domains. In addition to the other studies, Sliwinski et al. (2006) reported their results for episodic memory in raw data units (range: $0-72$; preterminal decline: 0.77 raw-score units per year; terminal decline: 1.42 raw-score units per year).
} 
decline should begin, what form the transition takes (gradual vs. abrupt), and what might explain interindividual differences in the timing of the transition. In general, the innovations made on the modeling side now require further precision in the theory of terminal decline.

Although the methodological articulations of terminal decline are at the limits of what is currently possible with the data at hand, it must be noted that the present examination remains descriptive and does not allow for causal inferences. The modeling approach used mortality as an independent variable and examined, in a post hoc manner, changes that occurred prior to known deaths. Models operationalizing predictive theories, however, would require the death date to be unknown - using observed accelerations in decline to predict subsequent transition into the death state (for one possible avenue, see Ghisletta et al., 2006). Independent of modeling considerations, it remains an open conceptual question as to whether psychosocial factors convey mortality risks on their own or whether they reflect the effects of pathologic processes. Regarding well-being, for example, one position argues that selfevaluations of one's life and aging do have physiological effects on cardiovascular and immune functioning (Danner et al., 2001; Pressman \& Cohen, 2005) that may have long-term effects on functioning and survival. Another position argues that well-being ratings might represent evaluations that reflect quite accurate summary perceptions of an individual's level and change in functioning in a variety of other domains that are more directly linked to mortality (cf. Maier \& Smith, 1999). Reports from the Berlin Aging Study, however, suggest that interindividual differences in the extent of terminal change in life satisfaction may not be accounted for by comorbidities or correspondent terminal decline in various aspects of cognitive functioning (Gerstorf et al., 2008). Unfortunately, we were unable to examine such questions with the data at hand (i.e., the SOEP is primarily an economic study). Examining these and other etiological questions, however, would shed some initial light on potentially underlying mechanisms. For example, it is conceivable that various causes of death (e.g., cerebrovascular, cardiac, and cancer) and the conditions associated with the process of dying (e.g., institutionalization, frailty) may account for differential portions of individual differences in terminal decline of well-being. In sum, there is much further work to be done, some of which will require more causally oriented designs and methods.

To conclude, evidence has been building that events such as marriage and unemployment systematically drive lasting changes in individuals' life satisfaction (e.g., Lucas et al., 2003). The present study adds to these notions, suggesting that impending death may represent another factor that contributes to a set of systematic changes in life satisfaction, albeit ones that lead up to the event rather than away from it. We found evidence of structured terminal decline and along with other recent studies provide further evidence that proximity to death is associated with substantial losses not only in "age-sensitive domains," such as intellectual and sensory functioning, but also in relatively "ageinsensitive" domains, such as well-being, that are usually well preserved into old and advanced old age (see also Gerstorf et al., 2008; Mroczek \& Spiro, 2005). As more and more data on the last years of life become available across multiple domains of functioning, and the analytical techniques become more refined, there is no doubt that more will be learned about how and why mortality-related processes contribute to the experiences of late life-steps along the way to the greater goal of doing what we can to make the terminal years ones filled with the satisfaction of living a good life.

\section{References}

Bäckman, L., \& MacDonald, S. W. S. (2006). Death and cognition: Synthesis and outlook. European Psychologist, 11, 224-235.

Baltes, P. B. (2006). Facing our limits: Human dignity in the very old Daedalus, 135, 33-39.

Baltes, P. B., \& Labouvie, G. V. (1973). Adult development of intellectual performance: Description, explanation, modification. In C. Eisdorfer \& M. P. Lawton (Eds.), The psychology of adult development and aging (pp. 157-219). Washington, DC: American Psychological Association.

Baltes, P. B., \& Smith, J. (2003). New frontiers in the future of aging: From successful aging of the young old to the dilemmas of the fourth age. Gerontology, 49, 123-135.

Birren, J. E., \& Cunningham, W. R. (1985). Research on the psychology of aging: Principles, concepts and theory. In J. E. Birren \& K. W. Schaie (Eds.), Handbook of the psychology of aging (2nd ed., pp. 3-34). New York: Van Nostrand Reinhold.

Bosworth, H. B., \& Schaie, K. W. (1999). Survival effects in cognitive function, cognitive style, and sociodemographic variables in the Seattle Longitudinal Study. Experimental Aging Research, 25, 121-139.

Brockmann, H., \& Klein, T. (2004). Love and death in Germany: The marital biography and its effect on mortality. Journal of Marriage and Family, 66, 567-581.

Burkhauser, R. V., Giles, P., Lillard, D. R., \& Schwarze, J. (2005). Until death do us part: An analysis of the economic well-being of widows in four countries. Journals of Gerontology, Series B: Psychological Sciences and Social Sciences, 60, S238-S246.

Busse, E. W. (1969). Theories of aging. In E. W. Busse \& E. Pfeiffer (Eds.), Behavior and adaptation in late life (pp. 11-32). Boston: Little \& Brown.

Charles, S. T., Reynolds, C. A., \& Gatz, M. (2001). Age-related differences and change in positive and negative affect over 23 years. Journal of Personality and Social Psychology, 80, 136-151.

Crimmins, E. M. (2001). Mortality and health in human life spans. Experimental Gerontology, 36, 885-897.

Cudeck, R., \& Harring, J. R. (2007). Analysis of nonlinear patterns of change with random coefficient models. Annual Review of Psychology, 58, 615-637.

Cudeck, R., \& Klebe, K. J. (2002). Multiphase mixed-effects model for repeated measure data. Psychological Methods, 7, 41-63.

Danner, D. D., Snowdon, D. A., \& Friesen, W. V. (2001). Positive emotions in early life and longevity: Findings from the Nun Study. Journal of Personality and Social Psychology, 80, 804-813.

Diener, E., \& Diener, C. (1996). Most people are happy. Psychological Science, 7, 181-185.

Diener, E., Lucas, R. E., \& Scollon, C. N. (2006). Beyond the hedonic treadmill: Revising the adaptation theory of well-being. American Psychologist, 61, 305-314.

Fujita, F., \& Diener, E. (2005). Life satisfaction set point: Stability and change. Journal of Personality and Social Psychology, 88, 158-164.

Gerhardt, G. A., Cass, W. A., Yi, A., Zhang, Z., \& Gash, D. M. (2002). Changes in somatodendritic but not terminal dopamine regulation in aged rhesus monkeys. Journal of Neurochemistry, 80, 168-177.

Gerstorf, D., Lövdén, M., Röcke, C., Smith, J., \& Lindenberger, U. (2007). Well-being affects changes in perceptual speed in advanced old age: Longitudinal evidence for a dynamic link. Developmental Psychology, 43, 705-718.

Gerstorf, D., Ram, N., Röcke, C., Lindenberger, U., \& Smith, J. (2008). Decline in life satisfaction in old age: Longitudinal evidence for links to distance-to-death. Psychology and Aging, 23, 154-168. 
Ghisletta, P., McArdle, J. J., \& Lindenberger, U. (2006). Longitudinal cognition-survival relations in old and very old age: 13-year data from the Berlin Aging Study. European Psychologist, 11, 204-223.

Haisken-De New, J. P., \& Frick, R. (2006). Desktop companion to the German Socio-Economic Panel Study (SOEP). Berlin, Germany: German Institute for Economic Research.

Hall, C. B., Lipton, R. B., Sliwinski, M. J., \& Stewart, W. F. (2000). A change point model for estimating the onset of cognitive decline in preclinical Alzheimer's disease. Statistics in Medicine, 19, 1555-1566.

Hertzog, C., \& Nesselroade, J. R. (2003). Assessing psychological change in adulthood: An overview of methodological issues. Psychology and Aging, 18, 639-657.

Infratest Sozialforschung. (2002). Verbesserung der Datengrundlagen für Mortalitäts- und Mobilitätsanalysen: Verbleibstudie bei Panelausfällen im SOEP [Improving the database for analyses of mortality and mobility: Examining panel attrition in the SOEP study]. Munich, Germany: http://www.diw.de/deutsch/sop/service/doku/docs/verbleibstudie.pdf

Kleemeier, R. W. (1962). Intellectual changes in the senium. Proceedings of the Social Statistics Section of the American Statistical Association, 1, 290-295.

Kroh, M. (2006). An experimental evaluation of popular well-being measures (Discussion Paper No. 546). Berlin, Germany: German Institute for Economic Research.

Kroh, M., \& Spie $\beta$, M. (2006). Documentation of sample sizes and panel attrition in the Socio-Economic Panel (SOEP), 1984-2005 (DIW Data Documentation No. 15). Berlin, Germany: German Institute for Economic Research.

Kunzmann, U., Little, T. D., \& Smith, J. (2000). Is age-related stability of subjective well-being a paradox? Cross-sectional and longitudinal evidence from the Berlin Aging Study. Psychology and Aging, 15, 511526.

Levy, B. R., Slade, M. D., Kunkel, S. R., \& Kasl, S. V. (2002). Longevity increased by positive self-perceptions of aging. Journal of Personality and Social Psychology, 83, 261-270.

Littell, R. C., Miliken, G. A., Stoup, W. W., \& Wolfinger, R. D. (1996). SAS system for mixed models. Cary, NC: SAS Institute.

Little, R. J. A., \& Rubin, D. B. (1987). Statistical analysis with missing data. New York: Wiley.

Lucas, R. E., Clark, A. E., Georgellis, Y., \& Diener, E. (2003). Reexamining adaptation and the set point model of happiness: Reactions to changes in marital status. Journal of Personality and Social Psychology, $84,527-539$.

Lucas, R. E., \& Gohm, C. (2000). Age and sex differences in subjective well-being across cultures. In E. Diener \& E. M. Suh (Eds.), Culture and subjective well-being (pp. 291-317). Cambridge, MA: MIT Press.

Maier, H., \& Smith, J. (1999). Psychological predictors of mortality in old age. Journals of Gerontology, Series B: Psychological Sciences and Social Sciences, 54B, P44-P54.

McArdle, J. J., \& Nesselroade, J. R. (2003). Growth curve analysis in contemporary psychological research. In J. Shinka \& W. Velicer (Eds.), Comprehensive handbook of psychology: Vol. 2. Research methods in psychology (pp. 447-480). New York: Wiley.

Mroczek, D. K., \& Kolarz, C. M. (1998). The effect of age on positive and negative affect: A developmental perspective on happiness. Journal of Personality and Social Psychology, 75, 1333-1349.

Mroczek, D. K., \& Spiro, A., III. (2005). Change in life satisfaction during adulthood: Findings from the Veterans Affairs Normative Aging Study. Journal of Personality and Social Psychology, 88, 189-202.

Palmore, E., \& Cleveland, W. (1976). Aging, terminal decline, and terminal drop. Journal of Gerontology, 31, 76-81.

Pressman, S. D., \& Cohen, S. (2005). Does positive affect influence health? Psychological Bulletin, 131, 925-971.
Ram, N., \& Grimm, K. J. (2007). Using simple and complex growth models to articulate developmental change: Matching method to theory. International Journal of Behavioral Development, 31, 303-316.

Raudenbush, S. W., \& Bryk, A. S. (2002). Hierarchical linear models: Applications and data analysis methods (2nd ed.). Thousand Oaks, CA: Sage.

Rendtel, U. (1995): Lebenslagen im Wandel: Panelausfälle und Panelrepräsentativität [Changing lives: Panel drop-out and panel representativeness]. New York: Campus.

Riegel, K. F., \& Riegel, R. M. (1972). Development, drop, and death. Developmental Psychology, 6, 306-419.

Schilling, O. (2006). Development of life satisfaction in old age: Another view on the paradox. Social Indicator Research, 75, 241-271.

Schimmack, U., Schupp, J., \& Wagner, G. G. (in press). The influence of environment and personality on the affective and cognitive component of subjective well-being. Social Indicator Research.

Schräpler, J.-P. (2004). Respondent behavior in panel studies. Sociological Methods and Research, 33, 118-156.

Siegler, I. C. (1975). The terminal drop hypothesis: Fact or artifact? Experimental Aging Research, 1, 169-185.

Singer, J. D., \& Willett, J. B. (2003). Applied longitudinal data analysis: Modeling change and event occurrence. New York: Oxford University Press.

Sliwinski, M. J., Stawski, R. S., Hall, R. B., Katz, M., Verghese, J., \& Lipton, R. B. (2006). On the importance of distinguishing pre-terminal and terminal cognitive decline. European Psychologist, 11, 172-181.

Smith, J., \& Gerstorf, D. (2004). Aging differently: Potentials and limits. In S. O. Daatland \& S. Biggs (Eds.), Ageing and diversity: Multiple pathways and cultural migrations (pp. 13-28). Bristol, United Kingdom: Policy Press.

Snijders, T. A. B., \& Bosker, R. J. (1999). Multilevel analysis: An introduction to basic and advanced multilevel modeling. London, United Kingdom: Sage.

Spiegelhalter, D. J., Thomas, A., Best, N. G., \& Lunn, D. (2007). WinBUGS Version 1.4.1 user manual [Computer software and manual]. Cambridge, United Kingdom: Medical Research Council Biostatistics Unit. Available from http://www.mrc-bsu.cam.ac.uk/bugs

Thorvaldsson, V., Hofer, S. M., \& Johansson, B. (2006). Aging and late-life terminal decline in perceptual speed. European Psychologist, 11, 196-203.

Wagner, G. G., Frick, J. R., \& Schupp, J. (2007). Enhancing the power of household panel studies: The case of the German Socio-Economic Panel Study (SOEP). Schmollers Jahrbuch, 127, 139-169.

Wang, L., \& McArdle, J. J. (2008). A simulation study comparison of Bayesian estimation with conventional methods for estimating unknown change points. Structural Equation Modeling, 15, 52-74.

White, N., \& Cunningham, W. R. (1988). Is terminal drop pervasive or specific? Journals of Gerontology, Series B: Psychological Sciences and Social Sciences, 43, P141-P144.

Wilson, R. S., Beck, T. L., Bienias, J. L., \& Bennett, D. A. (2007). Terminal cognitive decline: Accelerated loss of cognition in the last years of life. Psychosomatic Medicine, 69, 131-137.

Wilson, R. S., Beckett, L. A., Bienias, J. L., Evans, D. A., \& Bennett, D. A. (2003). Terminal decline in cognitive function. Neurology, 60, 17821787.

Zhang, Z., Hamagami, F., Wang, L., Grimm, K. J., \& Nesselroade, J. R. (2007). Bayesian analysis of longitudinal data using growth curve models. International Journal of Behavioral Development, 31, 374-383.

Received June 28, 2007

Revision received April 1, 2008 Accepted April 8, 2008 